\title{
Artigo Original / Original Paper \\ Physciaceae foliosas do Parque Estadual da Cantareira, estado de São Paulo. III. Espécies do Gênero Physcia
}

Foliose Physciaceae from the Parque Estadual da Cantareira, São Paulo state. III. Species of the genus Physcia

Michel Navarro Benatti ${ }^{1,2,3}$ \& Marcelo Pinto Marcelli ${ }^{1}$

\begin{abstract}
Resumo
Este trabalho apresenta dados obtidos a partir do levantamento de espécimes de fungos liquenizados de hábito folioso coletados no Parque Estadual da Serra da Cantareira até o final da década de 1990 e depositados atualmente no herbário SP. Foram encontradas e analisadas amostras de seis espécies de fungos liquenizados pertencentes ao gênero Physcia da família Physciaceae. São apresentadas descrições, comentários, ilustrações e uma chave para as espécies encontradas.
\end{abstract}

Palavras-chave: Ascomycota, fungos, liquens, Mata Atlântica.

\begin{abstract}
This survey includes data obtained from foliose lichens specimens collected at Parque Estadual da Serra da Cantareira until the end of the 1990s, and currently deposited in SP herbarium. Samples of six foliose lichen species belonging to the genus Physcia of the family Physciaceae were found and analyzed. Descriptions, comments, illustrations and a key for the reported species are presented.
\end{abstract}

Key words: Ascomycota, fungi, lichens, Atlantic Forest.

\section{Introdução}

Este trabalho integra o levantamento das espécies de Physciaceae foliosas encontradas no Parque Estadual da Cantareira, SP, iniciado com o estudo das espécies de Dirinaria (Tuck.) Clem., Hyperphyscia Müll. Arg. e Pyxine Fr. (Benatti \& Jungbluth 2014) e continuado com as espécies de Heterodermia Trevis e Polyblastidium Kalb (Benatti \& Marcelli 2017). O gênero Physcia (Schreb.) Michaux é um dos seis gêneros de espécies com talos de hábito folioso de Physciaceae encontrados no Brasil (Marcelli 2011; Jungbluth \& Marcelli 2012), conforme mencionados por Benatti \& Jungbluth (2014), e embora a família seja relativamente pouco estudada no neotrópico, tratase de um dos gêneros mais expressivos da família em número de espécies na região, atrás apenas de Heterodermia Trevis.
São conhecidas até o momento 71 espécies de Physcia no mundo das quais 27 ocorrem no Brasil. Espécies de Physcia apresentam tipicamente atranorina no córtex superior e medula branca, sendo que uma grande parte das espécies que ocorrem no Brasil tem a superfície inferior escurecida e contêm atranorina $(\mathrm{K}+$ amarelo) na medula (Jungbluth \& Marcelli 2012). Os apotécios apresentam sempre um excípulo talino, diferentemente do que é observado em Pyxine, e não há ocorrência de uma substância no epitécio que reaja $\mathrm{K}+$ púrpura tal como ocorrem em espécies de Dirinaria e Pyxine; difere de Hyperphyscia principalmente pela ausência de atranorina cortical e pelos conídios filiformes muito mais longos nas espécies deste gênero. Os ascósporos de Physcia são do tipo Pachysporaria (Malme) M.Choisy (Jungbluth \& Marcelli 2012).

\footnotetext{
'Instituto de Botânica de São Paulo, Núcleo de Pesquisa em Micologia, Av. Miguel Estéfano 3687, Água Funda, São Paulo, SP, Brasil.

${ }^{2}$ ORCID: https://orcid.org/0000-0002-2625-4194

${ }^{3}$ Autor para correspondência: michel_benatti@yahoo.com.br
} 
Dados sobre a localidade de estudo dos liquens no Parque Estadual da Serra da Cantareira e arredores podem ser consultados em Benatti (2012). Até o momento, além do estudo com outros cinco gêneros de Physciaceae foliosas mencionados (Benatti \& Jungbluth 2014; Benatti \& Marcelli 2017), foram publicadas como outras partes do levantamento de liquens foliosos para a localidade as pequenas espécies de Parmeliaceae ciliadas (Benatti 2012), as espécies eciliadas dos gêneros Canoparmelia Elix \& Hale e Crespoa Lendemer \& Hodkinson (Benatti 2014b), os cianoliquens foliosos (Benatti et al. 2013), e as espécies de Parmotrema Massalongo (Benatti 2013, 2014a).

O objetivo deste trabalho foi realizar o levantamento das espécies do gênero Physcia da família Physciaceae do Parque Estadual da Cantareira, sendo que a maioria das espécies encontradas foi descrita somente a partir da segunda metade da década de 1980. São fornecidos uma chave de identificação, descrições comentadas, dados das localidades e de ambientes para as espécies encontradas.

\section{Material e Métodos}

A metodologia de coleta para fungos liquenizados foliosos é descrita em Hale (1987), Malcolm \& Galloway (1997) e Benatti \& Marcelli (2007). As principais características analisadas nos gêneros aqui estudados são as apontadas em Jungbluth \& Marcelli (2012).

O material coletado na área da Reserva da Serra da Cantareira e depositado no herbário Maria Eneyda P. Kauffmann Fidalgo (SP) do Instituto de Botânica foi identificado conforme a metodologia tradicional, descrita em Hale (1979) e Galloway $(1985,2007)$. As análises morfológicas consistem na observação de características macro e microscópicas das estruturas vegetativas e de reprodução, mediante uso de estereomicroscópio e microscópio óptico.

A metodologia de análises químicas consistiu em testes de coloração, fluorescência à luz UV, e cromatografia em camada delgada (CCD) com solvente C, seguindo Asahina \& Shibata (1954), Walker \& James (1980), White \& James (1985), Huneck \& Yoshimura (1996) e Orange et al. (2001). As substâncias liquênicas detectadas foram conferidas e confirmadas pela Dra. Neli K. Honda, da UFMS.

A bibliografia taxonômica utilizada incluiu Aptroot (1987, 1988, 2001, 2002), Aptroot \& Sipman (1989), Aptroot \& Seaward (1999), Aptroot et al. (2002, 2007), Brodo et al. (2001), Calvelo \& Liberatore (2002), Esslinger \& Egan (1995), Fleig (1988), Fleig \& Grüninger (2000a,b), Galloway \& Moberg (2005), Harris (1990), Hansen et al. (2008), Jungbluth (2010), Käffer \& Mazzitelli (2005), Krog (2000), Lisboa (1952), Llimona \& Hladun (2001), Lynge (1924), Marcano et al. (1996), McCarthy \& Elix (2002), Marcelli (1991, 1998), Mazzitelli et al. (1999), Moore (1968), Moberg (1986, 1989, 1990, 1997, 2001, 2002, 2004), Osorio (1970, 1977, 1981, 1992), Osorio et al. (1997), Pereira \& Marcelli (1989), Scutari (1992, 1995), Sipman \& Wolf (1998), Swinscow \& Krog (1988), Tenorio et al. (2002), Thomson (1963), Vainio (1890), WietzkeBeckenkamp \& Pereira (1997), Wolseley et al. (2002) e Zahlbruckner (1909).

\section{Resultados e Discussão}

Foram encontradas ao todo seis espécies pertencentes ao gênero Physcia. É apresentada uma chave de identificação para as espécies, assim como a descrição e comentários sobre as espécies encontradas.

\section{Chave de identificação das espécies de Physcia do Parque Estadual da Cantareira}

1. Talo sorediado.

2. Sorais marginais lineares interrompidos, em forma de crescente ou labriformes; lado de baixo predominantemente marrom e estriado, todo corticado exceto nas porções distais; córtex inferior prosoplectenquimático 2. Physcia atrostriata

2'. Sorais principalmente laminais; lado de baixo marrom a enegrecido não estriado, uniformemente corticado até as porções distais; córtex inferior paraplectenquimático.

3. Sorais laminais, secundariamente chegando até as margens 4

4. Lacínias ca. 0,5-1 mm larg.; sorais distintos e esparsos formados a partir de rupturas no córtex; lado de baixo marrom claro a marrom (negro quando amadurecido?)..... 
4'. Lacínias ca. 1-2(-3) mm larg.; sorais estritamente laminais, inicialmente delimitados, orbiculares e crateriformes, tornando-se gradativamente mais densos; lado de baixo cinza amarronzado a marrom escuro..... 4. Physcia krogiae

3'. Sorais marginais, secundariamente laminais, irregulares e aglomerados; lado de baixo marrom escuro a enegrecido, com as bordas mais claras 6. Physcia sorediosa

1'. Talo não sorediado.

5. Talo lobulado; córtex inferior paraplectenquimático, marrom a marrom escuro com apenas as margens brancas. 5. Physcia lobulata

5'. Talo não lobulado; córtex inferior prosoplectenquimático, branco a creme claro.

1. Physcia alba

1. Physcia alba Müll. Arg., Rev. Mycol. 9: 136. 1887.

Fig. 1a-b

Talo cinza esbranquiçado, de até $3,0 \mathrm{~cm}$ diâm., corticícola. Lacínias 0,3-0,9 mm larg., planas a sutilmente côncavas ou convexas, \pm adnatas e adpressas, não pruinosas, contíguas a parcialmente imbricadas, de ramificação irregular a \pm subdicotômica, eciliados, ápices truncados a subtruncados, planos a sutilmente côncavos, as margens sinuosas a subirregulares, planas, córtex superior contínuo, liso, paraplectenquimático. Máculas ausentes. Isídios, sorédios e pústulas ausentes. Camada de algas contínua. Medula totalmente branca, sem trechos pigmentados. Lado de baixo corticado, do centro às bordas das lacínias, branco a creme claro, lustroso, liso a ocasionalmente subrugoso, sem venações, as margens lisas, lustrosas, às vezes de coloração cinza amarronzada clara, córtex inferior prosoplectenquimático. Rizinas $0,2-0,5 \mathrm{~mm}$ compr., concoloridas ao córtex ou raramente amarronzadas, geralmente simples a raramente irregularmente ramificadas, frequentes, distribuídas homogeneamente. Apotécios abundantes, 0,3-1,3 mm diâm., planos a subcôncavos, sésseis a adnatos, laminais, margens e anfitécios lisos às vezes tornando-se subrugosos quando velhos, sem ornamentações, disco enegrecido, epruinoso. Ascósporos transversalmente septados com um septo, inicialmente hialinos gradualmente tornando-se pigmentados (marrons), do tipo Pachysporaria, 21-28 × 8-11 $\mu \mathrm{m}$. Picnídios não encontrados.

O teste de coloração indica córtex $\mathrm{K}+$ amarelo, $\mathrm{UV}$ - (atranorina); medula $\mathrm{K}+$ amarelo, $\mathrm{C}-, \mathrm{KC}-$, $\mathrm{P}-, \mathrm{UV}-$ (atranorina, zeorina e triterpenóides indeterminados).

Distribui-se na América do Norte e América do Sul (Aptroot 2002; Fleig \& Grüninger 2000a; Jungbluth 2010; Mazzitelli et al. 1999; Moore 1968; Moberg 1990, 2002; Osorio 1981, 1992;
Osorio et al. 1997; Scutari 1992, 1995; Thomson 1963; Vainio 1890; Wietzke-Beckenkamp \& Pereira 1997; Zahlbruckner 1909).

Material estudado: Mairiporã, sobre tronco de árvore em mata de encosta próximo a um pomar com clareiras rochosas, II.1981, M.P. Marcelli 16904, 16907 (SP).

De acordo com Jungbluth (2010), é relativamente semelhante a $P$. tenuis e a $P$. kalbii, porém a espécie tem lado de baixo branco a creme (e apresenta reação $\mathrm{K}+$ amarela indicando a presença de zeorina, o que não ocorre em $P$. tenuis). O lado de baixo é branco, e não marrom ou negro como descrito para $P$. kalbii. A largura das lacínias também está de acordo com Moberg (1990) e a variação é ligeiramente mais estreita que a mais larga mencionada por Jungbluth (2010). Além da coloração característica do córtex inferior, $P$. alba não apresenta rizinas retrorsas ou cílios nos apotécios como citados para $P$. kalbii, sendo que mesmo os apotécios mais desenvolvidos não têm nenhum cílio.

As informações em Jungbluth (2010) são conflitantes quanto à anatomia do córtex inferior, ora referindo-se você como prosoplectenquimático (chave de identificação), ora como paraplectenquimático (descrição) sendo que nos comentários é caracterizado como paraplectenquimatico, mas enquanto outras espécies são diferidas por serem paraplectenquimáticas. Nosso material está de acordo com Moberg (1990) que cita o córtex inferior como prosoplectenquimático.

As lacínias são bem estreitas, menores que os descritos por exemplo para o material tipo por Jungbluth (2010); entretanto, eles tem quase o mesmo tamanho visto nos espécimes de Physcia erumpens. De acordo com Moberg (1990), embora as lacínias alcancem 1,5 mm de largura elas são "normalmente mais estreitas", o que nos leva a considerar que é a variação mais comumente encontrada. Os ascósporos em nosso 

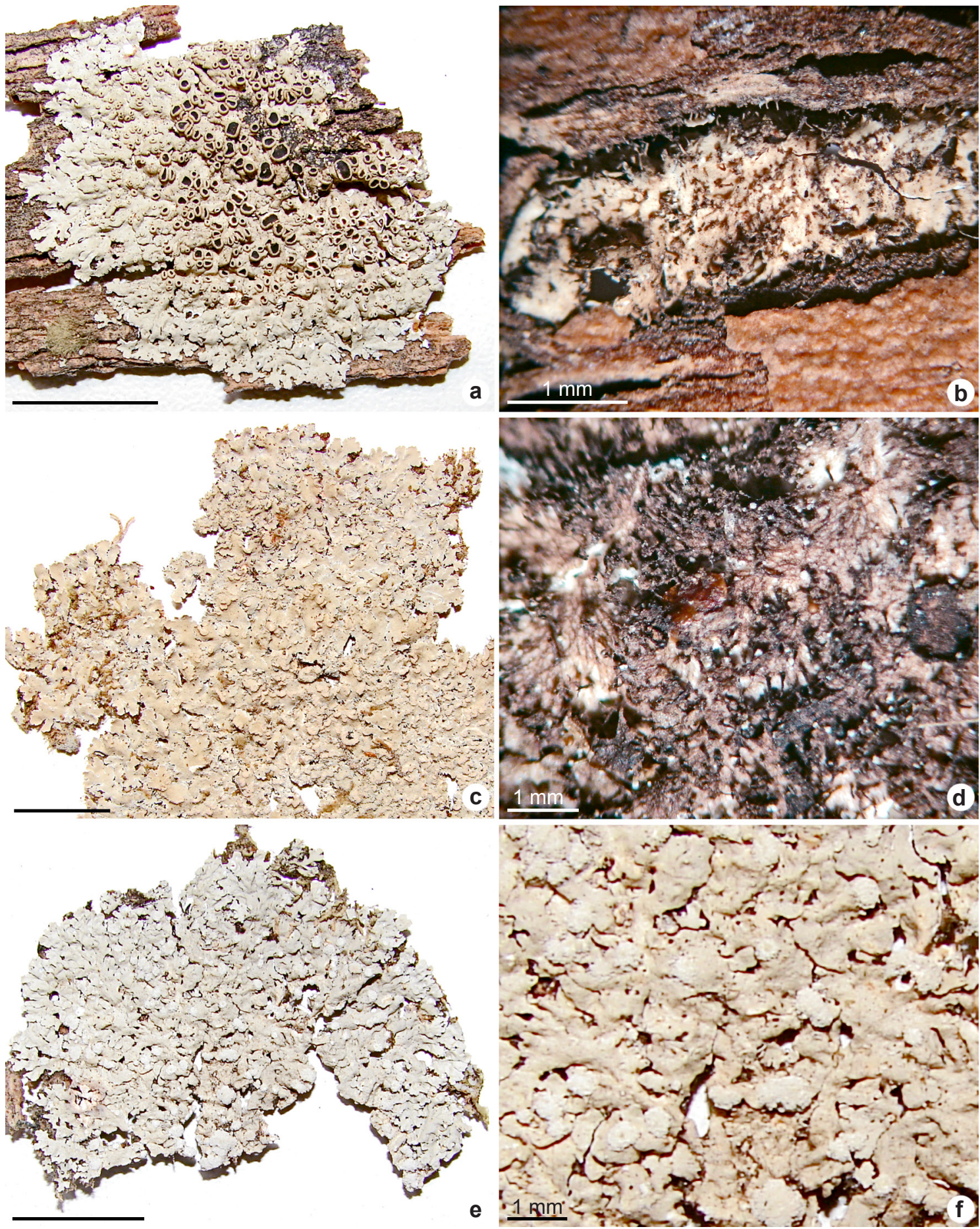

Figura 1 - a. Physcia alba; b. detalhe do lado de baixo em P. alba; c. Physcia atrostriata; d. detalhe do lado de baixo em P. atrostriata; e. Physcia erumpens; f. detalhe dos sorais em P. erumpens. Barras de escala: $1 \mathrm{~cm}$, exceto onde indicado. (a. Marcelli 16907; c. Marcelli 13450; e. Marcelli 13401).

Figure 1 - a. Physcia alba; b. detail ofn the lower surface of $P$. alba; c. Physcia atrostriata; d. detail of the lower surface of P. atrostriata; e. Physcia erumpens; f. detail of the soralia of P. erumpens. Scale bars: $1 \mathrm{~cm}$, except where indicated. (a. Marcelli 16907; c. Marcelli 13450; e. Marcelli 13401). 
material também estão dentro das medidas dadas por Moberg (1990), sendo que o grupo de espécies que inclui $P$. erumpens e $P$. alba aparenta ter os ascósporos dentro da faixa $20-30 \times 8-12 \mu \mathrm{m}$.

\section{Physcia atrostriata Moberg, Nordic Journal of} Botany 6: 853. 1986.

Fig. 1c-d

Talo cinza esbranquiçado claro, de até $6,5 \mathrm{~cm}$ diâm., corticícola ou muscícola. Lacínias 0,9-2,2 mm larg., planas a sutilmente convexas, \pm adnatas a sutilmente ascendentes e pouco adpressas, com pruína de intensidade e distribuição irregular (ausente a presente) tanto nas partes distais como proximais, imbricadas a sobrepostas, de ramificação irregular a dicotômica, eciliados, ápices subtruncados a arredondados, planos a \pm côncavos, as margens irregularmente incisas, acentuadamente mais sinuosas conforme se tornam sorediados, córtex superior contínuo, liso, paraplectenquimático. Máculas ausentes. Isídios e pústulas ausentes. Sorais lineares interrompidos, em forma de crescentes, ou labriformes irregulares, principalmente marginais por vezes galgando a submargem tornando-se gradativamente mais abundantes nas partes velhas, raros laminais irregulares e restritos a porções mais distais, de coloração branca, sorédios farinhosos. Camada de algas contínua. Medula totalmente branca, sem trechos pigmentados. Lado de baixo corticado na maior parte, exceto nas porções distais inferiores das lacínias, o centro corticado uniformemente marrom, às vezes escuro ou enegrecido, pouco lustroso, liso a venado, as partes brancas distais comumente decorticadas a partir dos ápices, lisas a venadas, opacas, as venações ocasionalmente tornando-se mais escuras e estendendo-se a partir do córtex inferior originando rizinas, córtex inferior prosoplectenquimático. Rizinas $0,2-0,8$ $(-1,2) \mathrm{mm}$ compr., concoloridas ao córtex ou mais escurecidas, raro esbranquiçadas junto aos ápices, comumente simples ou em parte irregularmente ramificadas, frequentes, tornando-se mais comuns em partes distais de onde surgem parcialmente a partir das venações, distribuídas homogeneamente. Apotécios e picnídios não encontrados.

$\mathrm{O}$ teste de coloração indica córtex $\mathrm{K}+$ amarelo, $\mathrm{UV}$ - (atranorina); medula $\mathrm{K}+$ amarelo, $\mathrm{C}-, \mathrm{KC}-$, $\mathrm{P}-$, UV- (atranorina, zeorina, e triterpenóides indeterminados).

Tem distribuição pantropical, estendendo-se a regiões subtropicais (Aptroot 1987, 1988, 2001; Aptroot \& Sipman 1989; Aptroot \& Seaward 1999; Aptroot et al. 2007; Brodo et al. 2001; Calvelo \&
Liberatore 2002; Esslinger \& Egan 1995; Galloway \& Moberg 2005; Harris 1990; Hansen et al. 2008; Jungbluth 2010; Krog 2000; Llimona \& Hladun 2001; Marcano et al. 1996; McCarthy \& Elix 2002; Moberg 1986, 1989, 1990, 1997, 2001, 2004; Scutari 1992, 1995; Sipman \& Wolf 1998; Swinscow \& Krog 1988; Tenorio et al. 2002).

Material estudado: Mairiporã, Serra da Cantareira, em tronco de árvore derrubado na construção de condomínio, 11.III.1989, M.P. Marcelli 6077 (SP). São Paulo, Parque Estadual da Cantareira, próximo ao Lago das Carpas, sobre tronco de árvore na mata, 25.VI.1991, M.P. Marcelli, A. Rezende \& O. Yano 11440 (SP); sobre tronco de árvore na mata, 25.VI.1991, M.P. Marcelli, O. Yano, A. Rezende, F.M.M. Coppolla 11552 (SP); sobre tronco de árvore no cruzamento Pé de Galinha, 25.VI.1991, M.P. Marcelli, O. Yano, A. Rezende, F.M.M. Coppolla 11561 (SP); sobre tronco de árvore em bosque, na sombra clara, 15.VII.1991, M.P. Marcelli \& A. Rezende 11802 p. max. p. (SP); na estrada principal para o Lago das Carpas, sobre tronco de arvoreta na mata, 30.III.1992, M.P. Marcelli, A. Rezende \& O. Yano 13416, 13450 (SP).

O lado de baixo em alguns espécimes é tão amarronzado que é quase impossível discernir as venações nas partes mais velhas; só podem ser vistas realmente distintas em porções mais jovens ou já junto a porção ecorticada dos ápices; é engraçado, mas em alguns talos com várias porções claras com venações marrons, lembrando de certa forma o aspecto das rizinas que se vê em Peltigera Willd.

Embora todos os materiais apresentem pruína cortical, aparentam ser muito irregulares quanto à intensidade e a distribuição. A pruína é mais facilmente visualizável em porções distais das lacínias, porém isso não significa que todas as lacínias contenham pruína.

Um dos espécimes é um pequeno talo muscícola (M.P.Marcelli 11440) que tem o mesmo aspecto geral dos demais, mas o lado de baixo se parece com o descrito por Jungbluth (2010) para Physcia crispa, ("branco a marfim, opaco, liso a levemente venado"), uma outra espécie sorediada de sorais marginais. É possivelmente devido ao estágio mais imaturo do material.

O recorte das margens é bastante irregular. Apenas quando sorediam mais densamente é que aparentam um aspecto de formato mais regular, sinuoso, como descrito por Jungbluth (2010).

Todos os espécimes estudados são cinza esbranquiçados, e sorais laminais são bem escassos, provavelmente surgidos de algumas quebras, havendo alguns poucos em poucos espécimes, restritos quase que sempre às partes distais das 
lacínias. Não há nenhum indício da formação contínua de sorais laminais em nenhum dos materiais.

\section{Physcia erumpens Moberg, Nord. J. Bot. 6: 856.} 1986.

Fig. 1e-f

Talo cinza esbranquiçado, de até $3,5 \mathrm{~cm}$ diâm., corticícola. Lacínias $0,3-0,8(-1,1) \mathrm{mm}$ larg., planas a sutilmente convexas, adnatas e adpressas, muito levemente pruinosas apenas nas porções distais, contíguas a parcialmente imbricadas, de ramificação irregular a dicotômica, eciliadas, ápices truncados a subarredondados, planos a sutilmente convexos, as margens lisas a sinuosas, planas, córtex superior contínuo, liso, paraplectenquimático. Máculas ausentes. Isídios e pústulas ausentes. Sorais laminais, formados por aberturas no córtex, orbiculares a capitados ou ocasionalmente oblongos, tornando-se crateriformes a partir da eliminação dos sorédios, normalmente distintos não formando aglomerações, sorédios farinhosos a granulares. Camada de algas contínua. Medula totalmente branca, sem trechos pigmentados. Lado de baixo corticado, do centro às bordas das lacínias, marrom claro a marrom, lustroso, liso a ocasionalmente subrugoso, sem venações, as margens lisas, lustrosas, córtex inferior paraplectenquimático. Rizinas $0,2-0,6 \mathrm{~mm}$ compr., concoloridas ao córtex, geralmente simples a raramente irregularmente ramificadas, frequentes a abundantes e por vezes aglutinadas, distribuídas homogeneamente. Apotécios e picnídios não encontrados.

$\mathrm{O}$ teste de coloração indica córtex $\mathrm{K}+$ amarelo, UV- (atranorina); medula $\mathrm{K}+$ amarelo, $\mathrm{C}-, \mathrm{KC}-$, $\mathrm{P}-, \mathrm{UV}-$ (atranorina, zeorina e triterpenóides indeterminados).

Tem distribuição pantropical, com citações para regiões subtropicais, exceto ainda para a Ásia (Aptroot 1988; Calvelo \& Liberatore 2002; Galloway \& Moberg 2005; Jungbluth 2010; Käffer \& Mazzitelli 2005; Marcano et al. 1996; Moberg 1989, 1990, 1997, 2004; Osorio 1992; Scutari 1990, 1995; Swinscow \& Krog 1988).

Material estudado: São Paulo, Parque Estadual da Cantareira, na estrada principal para o Lago das Carpas, sobre tronco de arvoreta isolada, 30.III.1992, M.P. Marcelli, A. Rezende \& O. Yano 13401 (SP).

Jungbluth (2010) mencionou um córtex inferior negro para esta espécie, de acordo com o que foi também dito por Moberg (1990), mas o único espécime encontrado na localidade de estudo é marrom no lado de baixo, bem parecido com os espécimes de sorais marginais de $P$. sorediosa neste aspecto, porém ainda mais claro. Embora Jungbluth (2010) tenha mencionado máculas fracas, não conseguimos visualizar nenhuma neste espécime também. Quase não há sinais de pruína no material. O córtex tem um brilho que faz ele parecer perolado. Há bem pouca pruína em alguns dos ápices das lacínias, e ainda sim bem difícil de se perceber.

Como todas as demais características estão em concordância e o talo é relativamente pequeno, suspeitamos que a cor clara e demais caracteres escassos como máculas e pruína possam talvez ser devido a um estágio mais inicial de maturação do próprio córtex inferior. Corrobora para isso o fato de que o lado de baixo deste espécime é predominantemente marrom claro, eventualmente tornando-se marrom mais escuro (de tom mediano) em parte do centro (que deveria estar gradualmente escurecendo), apesar de não haver áreas negras em trecho algum. Também não há uma distinção de coloração nítida entre margens e centro.

4. Physcia krogiae Moberg, Nord. J. Bot. 6: 858. 1986.

Fig. 2a-b

Talo cinza azulado claro, de até $8,5 \mathrm{~cm}$ diâm., corticícola. Lacínias $0,4-1,8(-3,1) \mathrm{mm}$ larg., planas a sutilmente côncavas, \pm adnatas e adpressas, levemente pruinosas nas porções distais, contíguas a ocasionalmente imbricadas, de ramificação irregular a subdicotômica, eciliadas, ápices subtruncados a subarredondados, planos a sutilmente convexos ou côncavos, as margens lisas a sinuosas ou irregulares, planas, córtex superior contínuo, liso a raramente subrugoso, paraplectenquimático. Máculas ausentes. Isídios e pústulas ausentes. Sorais laminais, orbiculares eventualmente adensando e tornandose confluentes, ocasionalmente soerguidos, às vezes estendendo-se à submargem, concoloridos a brancos, sorédios farinhosos a granulares. Camada de algas contínua. Medula totalmente branca, sem trechos pigmentados. Lado de baixo corticado, do centro às bordas das lacínias, cinza amarronzado a marrom escuro, pouco lustroso a opaco, liso a ocasionalmente subrugoso, sem venações, as margens lisas, lustrosas, frequentemente com uma fina linha branca na borda, córtex inferior paraplectenquimático. Rizinas $0,2-0,8 \mathrm{~mm}$ compr., concoloridas ao córtex, geralmente simples a raramente irregularmente ramificadas, às vezes aglutinadas, esparsas a frequentes, distribuídas homogeneamente. Apotécios e picnídios não encontrados. 

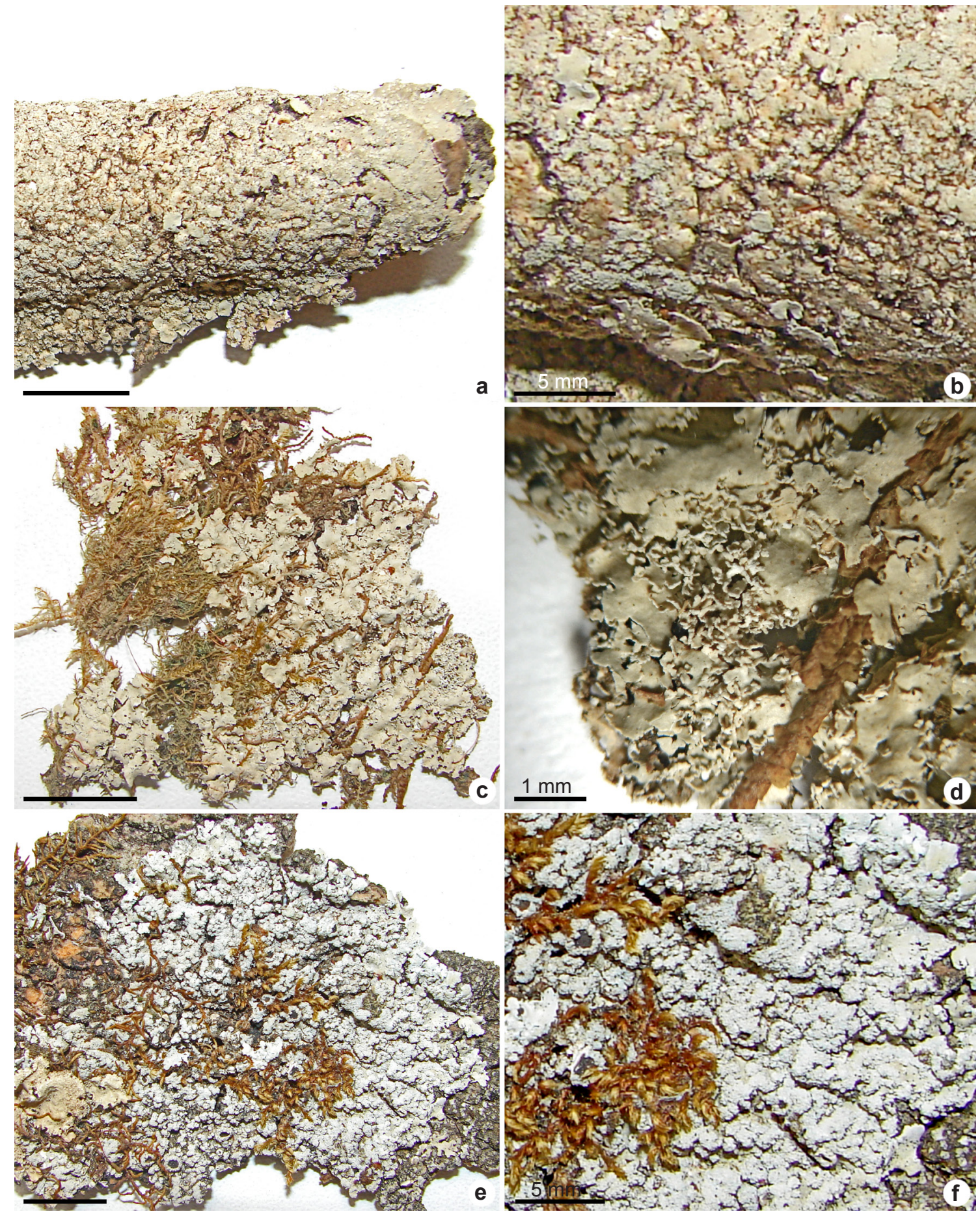

Figura 2 - a. Physcia krogiae; b. detalhe dos sorais em P. krogiae; c. Physcia lobulata; d. detalhe das lacínulas e lóbulos de P. lobulata; e. Physcia sorediosa; f. detalhe dos sorais em P. sorediosa. Barras de escala: $1 \mathrm{~cm}$, exceto onde indicado. (a. Marcelli 13561; c. Benatti 995; e. Marcelli 13571; f. Marcelli 13447).

Figure 2 - a. Physcia krogiae; b. detail of the soralia of $P$. krogiae; c. Physcia lobulata; d. detail of the lacinulae and lobules of $P$. lobulata; e. Physcia sorediosa; f. detail of the soralia of P. sorediosa. Scale bars: $1 \mathrm{~cm}$, except where indicated. (a. Marcelli 13561; c. Benatti 995; e. Marcelli 13571; f. Marcelli 13447). 
O teste de coloração indica córtex $\mathrm{K}+$ amarelo, UV- (atranorina); medula $\mathrm{K}+$ amarelo, $\mathrm{C}-, \mathrm{KC}-$, $\mathrm{P}-, \mathrm{UV}-$ (atranorina, zeorina e triterpenóides indeterminados).

Distribui-se na África e América do Sul (Aptroot 1988, 2001, 2002; Moberg 1986, 1990, 2004; Swinscow \& Krog 1988).

Material estudado: São Paulo, Serra da Cantareira, Parque Estadual da Cantareira, próximo ao lago perto da administração, sobre tronco de Lauraceae na beira do lago, 16.VII.1991, M.P. Marcelli, A. Rezende, O. Yano \& F.M.M. Coppolla 11780 (SP); núcleo da Pedra Grande, sobre tronco de arvoreta na sombra do bosque da Pedra Grande, 18.V.1992, M.P. Marcelli, A. Rezende, \& O. Yano $13561(\mathrm{SP})$.

É parecida com $P$. sorediosa, entretanto os sorais dos espécimes de $P$. sorediosa se agregam a tal ponto que chegam a revestirem o córtex superior, tornando difícil distinguir $P$. sorediosa de $P$. krogiae. Porém, é possível ver que a origem dos sorais é laminal em $P$. krogiae ao invés de marginal como em $P$. sorediosa, e em sua origem formam aglomerados pequenos e distintos, tais como mencionados por Jungbluth (2010).

Segundo Jungbluth (2010) as descrições de $P$. krogiae e $P$. sorediosa são bem parecidas, exceto pela largura das lacínias que em média é o dobro para P. krogiae, e pela disposição e formato dos sorais. As lacínias são comumente mais estreitas em relação à descritas em Jungbluth (2010), sendo em média tão estreitas como as do material de $P$. erumpens, embora uma parte delas que aparentam serem mais desenvoltas de fato alcancem a largura maior descrita em literatura (Moberg 1990; Jungbluth 2010). Os sorais parecem diferir para com os de P. erumpens também pelo fato de ficarem gradualmente maiores e mais densos.

O material de P. krogiae encontrado aqui não está fértil, portanto não foi possível verificar ascósporos.

O material encontrado na Cantareira não aparenta uma coloração azulada típica, possivelmente devido a herborização (talos de $P$. krogiae encontrados na natureza são distintamente azulados). Está mais para azul acinzentado, porém não de tom acentuado para o branco como ocorre em outras espécies de Physcia.

Não foram encontrados sinais de maculação em parte alguma, e como o córtex é densamente sorediado, mesmo cortes não deixam visualizações possíveis de cavidades abaixo que poderiam ser indícios. Já as partes distais das lacínias são frequentemente pruinosos. Jungbluth (2010) mencionou que maculações são raras.
5. Physcia lobulata Moberg, Nord. J. Bot. 10: 333. 1990.

Fig. 2c-d

Talo cinza esbranquiçado claro, de até 5,0 cm diâm., muscícola. Lacínias $0,3-0,7(-1,0)$ mm larg., \pm planas, adnatas e adpressas, não pruinosas, contíguas a parcialmente imbricadas, de ramificação irregular a dicotômica, eciliadas, ápices subtruncados a subarredondados, planos a sutilmente convexos, as margens sinuosas a crenadas ou irregulares, lacínulos, planos, córtex superior contínuo, liso, paraplectenquimático. Máculas ausentes. Isídios, sorédios e pústulas ausentes. Lacínulas presentes nas margens do talo, planas às vezes tornando-se um pouco ascendentes, simples a irregularmente ramificadas, $0,1-0,5 \times 0,1-0,3$ $\mathrm{mm}$. Camada de algas contínua. Medula totalmente branca, sem trechos pigmentados. Lado de baixo corticado do centro às bordas das lacínias, marrom a marrom escuro, pouco lustroso, liso a subrugoso, sem venações, as margens lisas a subrugosas, pouco lustrosas, marrons claras a brancas, córtex inferior paraplectenquimático. Rizinas $0,1-0,2(-0,3) \mathrm{mm}$ compr., concoloridas ao córtex, simples, escassas, distribuídas homogeneamente. Apotécios e picnídios não encontrados.

$\mathrm{O}$ teste de coloração indica córtex $\mathrm{K}+$ amarelo, UV- (atranorina); medula $\mathrm{K}+$ amarelo, $\mathrm{C}-, \mathrm{KC}-, \mathrm{P}-, \mathrm{UV}-$ (atranorina, zeorina, leucotilina e triterpenóides indeterminados).

Tem distribuição na América Central, América do Sul e África (Aptroot 2002; Moberg 1990).

Material estudado: São Paulo, Parque Estadual da Cantareira, sobre musgo em tronco de árvore na mata, VIII.2000, M.N. Benatti 995 (SP).

O material estudado se parece muito com o descrito por Jungbluth (2010), apenas as lacínulas aparentando serem ainda menores (Jungbluth (2010) citou medidas entre $0,5-1,0 \mathrm{~mm}$ ). As lacínias têm praticamente as mesmas medidas. O único espécime coletado é um talo muscícola.

6. Physcia sorediosa (Vain.) Lynge, Vid.-Selsk. Skrifter I. Mat.-Naturvar. kl. 16: 27.1924. Physcia integrata var. sorediosa Vain., Acta Soc. Fauna Fl. Fenn. 7: 142. 1890.

$\equiv$ Physcia fragilenses Zahlbr., Annals Cryptog. Exot. 1: 211. 1928.

Fig. 2e-f

Talo cinza esbranquiçado claro, de até 13,5 cm diâm., corticícola. Lacínias 1,0-1,9 mm larg., planas a sutilmente convexas, \pm adnatas e pouco adpressas, não pruinosas a levemente pruinosas de distribuição e intensidades irregulares, 
comumente imbricadas, de ramificação irregular, eciliadas, ápices subarredondados, \pm planos a côncavos ou convexos, as vezes ascendentes, as margens lisas a irregularmente incisas, elevadas e acentuadamente mais sinuosas conforme se tornam sorediadas, córtex superior contínuo, liso, paraplectenquimático. Máculas ausentes. Isídios e pústulas ausentes. Sorais inicialmente marginais galgando o córtex superior, gradativamente mais abundantes nas partes proximais a ponto de revestir o córtex, tornando-se laminais irregulares, muito aglomerados e às vezes soerguendo-se, de coloração acinzentada, sorédios farinhosos a subgranulares. Camada de algas descontinuada por projeções do córtex superior. Medula totalmente branca, sem trechos pigmentados. Lado de baixo corticado, porém com uma fina borda decorticada branca rente aos ápices nas porções distais inferiores das lacínias, marrom a marrom escuro ou enegrecido, pouco lustroso, liso a subrugoso, sem venações, as margens lisas, pouco lustrosas, córtex inferior paraplectenquimático. Rizinas $0,2-0,7(-1,1)$ mm compr., concoloridas ao córtex, comumente simples ou em parte irregularmente ramificadas, frequentes, distribuídas homogeneamente. Apotécios ausentes a abundantes, 0,5-2,3 mm diâm., subcôncavos a côncavos, adnatos, laminais a ocasionalmente submarginais, margens e anfitécios lisos gradualmente tornando-se ornamentados com sorais, disco marrom escuro a enegrecido, epruinoso. Ascósporos transversalmente septados, inicialmente hialinos gradualmente tornando-se pigmentados (marrons), do tipo Pachysporaria, (19-)24-35 × (7-)10-14 $\mu \mathrm{m}$. Picnídios não encontrados.

O teste de coloração indica córtex $\mathrm{K}+$ amarelo, $\mathrm{UV}$ - (atranorina); medula $\mathrm{K}+$ amarelo, $\mathrm{C}-$, $\mathrm{KC}-$, $\mathrm{P}-, \mathrm{UV}-$ (atranorina, zeorina e triterpenóides indeterminados).

Tem distribuição Pantropical (Aptroot 1987, 1988, 2002; Aptroot et al. 2002; Brodo et al. 2001; Fleig 1988; Fleig \& Grüninger 2000a,b; Hansen et al. 2008; Jungbluth 2010; Lisboa 1952; Lynge 1924; Marcelli 1991, 1998; Mazzitelli et al. 1999; Moberg 1986, 1990, 1997, 2001, 2002; Osorio 1970, 1977; Pereira \& Marcelli 1989; Scutari 1992; Swinscow \& Krog 1988; como P. fragilescens, Vainio 1890; Wolseley et al. 2002).

Material estudado: São Paulo, Parque Estadual da Cantareira, na estrada principal para o Lago das Carpas, sobre tronco de Cedrella na mata, 30.III.1992, M.P. Marcelli, A. Rezende \& O. Yano 13447 (SP); Núcleo da Pedra Grande, sobre tronco de árvore na beira do lago,
18.V.1992, M.P. Marcelli, A. Rezende \& O. Yano 13571, 13573, 13574 (SP).

A largura das lacínias tende a ser nitidamente menor que o comprimento, e as margens são bem paralelas. Como mencionado por Jungbluth (2010), o padrão parece-se bem com o de $P$. atrostriata. Jungbluth (2010) também mencionou que a camada de algas em $P$. atrostriata é contínua, uma diferença para $P$. sorediosa que pode variar de contínua a interrompida. No material de $P$. atrostriata é visualizada pruína com mais frequência, sendo que o material de $P$. sorediosa quase não apresenta pruína, possivelmente por ser mais densamente sorediado. Uma outra diferença é o cortex inferior, prosoplectenquimático em $P$. atrostriata e paraplectenquimatico em $P$. sorediosa.

Os espécimes contendo apotécios tem ascósporos grandes para o padrão do gênero, porém ainda maiores que os citados em Jungbluth (2010) e Moberg (1990), mas englobando as medições mencionadas por ambos os autores dentro da variação total vista aqui. Há ascos dispostos lado a lado em que se vê ascósporos com até o triplo do tamanho uns dos outros, o que fica mais evidente depois de uma ruptura quando podem ser visualizados para medições. Há ascósporos maduros de menores dimensões, vários deles já bastante pigmentados e de lúmen claramente definido. Da mesma forma, há ascósporos de tamanhos diferentes e maiores, ainda hialinos e com lumens quase que definidos.

Moberg (1990) e Jungbluth (2010) tratam $P$. fragilescens e $P$. sorediosa como sendo sinônimos, porém os ascósporos da primeira são descritos maiores que da segunda (o que colocaria nosso material mais próximo de $P$. fragilescens). Por exemplo, os ascósporos citados por Swinscow \& Krog (1988) para P. fragilescens estão bem mais próximos em tamanho aos vistos nos espécimes aqui descritos. A situação de sinonímia entre as duas espécies ainda aparenta requerer uma melhor definição, possivelmente com estudos moleculares envolvendo os materiais de esporos de tamanho díspar.

O material de $P$. sorediosa tem sorais predominantemente laminais - que chegam a revestir o cortex - e o lado de baixo é uniformemente marrom escuro, com as bordas das lacínias aparentando serem sutilmente decorticadas. Jungbluth (2010) mencionou que $P$. sorediosa é maculada, definindo a forma das máculas como claras e raramente distintas, as vezes abundantes em áreas proximais delimitadas, com aspecto de 
mancha a subreticulares. O material visto aqui é emaculado, o que poderia ser outra indicação de que $P$. fragilescens e $P$. sorediosa não sejam a mesma espécie. Jungbluth (2010) também descreveu que as margens são mais claras que o centro, sendo que nossos materiais não possuem uma distinção de cor nítida entre centro e margens.

\section{Agradecimentos}

$\mathrm{O}$ primeiro autor agradece à FAPESP (Fundação para o Amparo à Pesquisa do Estado de São Paulo), a concessão da bolsa de Iniciação Científica $n^{\circ}$ 00/01009-1. Os autores agradecem a Dra. Neli K. Honda, da UFMS, o auxílio com a cromatografia dos espécimes e confirmação das substâncias.

\section{Referências}

Aptroot A (1987) Pyxinaceae (Lichens). In: Rijn GARA (ed.) Flora of the Guyanas. Series E. Koeltz Scientific Books, Koenigstein. Pp. 1-53.

Aptroot A (1988) Lichens of Madagascar - the Pyxinaceae (Syn. Physciaceae). Cryptogamie Bryologie et Lichénologie 9: 141-147.

Aptroot A (2001) Lichens from Gambia, with a new black-fruiting isidiate Caloplaca on savannah trees. Cryptogamie, Mycologie 22: 265-270.

Aptroot A (2002) New and interesting lichens and lichenicolous fungi in Brazil. Fungal Diversity 9: 15-45.

Aptroot A \& Seaward MRD (1999) Annotated checklist of Hongkong lichens. Tropical Bryology 17: 57-101.

Aptroot A \& Sipman HJM (1989) New lichen records from the Philippines. Acta Bryolichenologica Asiatica 1: 31-41.

Aptroot A, Sparrius LB \& Lai MJ (2002) New Taiwan macrolichens. Mycotaxon 84: 281-292.

Aptroot A, Saipunkaew W, Sipman HJM, Sparrius LB \& Wolseley PA (2007) New lichens from Thailand, mainly microlichens from Chiang Mai. Fungal Diversity 24: 75-134.

Asahina Y \& Shibata S (1954) Chemistry of lichen substances. Japan Society for the Promotion of Science. Ueno, Tóquio. 240p.

Benatti MN (2012) Pequenas espécies de Parmeliaceae ciliadas no Parque Estadual da Cantareira, estado de São Paulo, Brasil: gêneros Bulbothrix, Parmelinella e Parmelinopsis (Parmeliaceae, Ascomycota). Hoehnea 39: 207-218.

Benatti MN (2013) Espécies de Parmotrema (Parmeliaceae, Ascomycota) no Parque Estadual da Cantareira, estado de São Paulo, Brasil. I. As espécies com máculas efiguradas ou reticulares. Hoehnea 40: 649-659.

Benatti MN (2014a) Espécies de Parmotrema
(Parmeliaceae, Ascomycota) no Parque Estadual da Cantareira, estado de São Paulo, Brasil. II. As espécies emaculadas ou com máculas irregulares. Hoehnea 41: 81-102.

Benatti MN (2014b) Pequenas espécies de Parmeliaceae (Ascomycota) eciliadas no Parque Estadual da Cantareira, estado de São Paulo, Brasil: gêneros Canoparmelia e Crespoa. Rodriguésia 65: 587-597.

Benatti MN \& Jungbluth P (2014) Physciaceae foliosas do Parque Estadual da Cantareira, estado de São Paulo I. Gêneros Dirinaria, Hyperphyscia e Pyxine. Iheringia 69: 17-27.

Benatti MN \& Marcelli MP (2007) Gêneros de fungos liquenizados dos manguezais do Sul-Sudeste do Brasil, com enfoque no manguezal do Rio Itanhaém, Estado de São Paulo. Acta Botanica Brasilica 21: 863-878.

Benatti MN \& Marcelli MP (2017) Physciaceae foliosas do Parque Estadual da Cantareira, estado de São Paulo II. Gêneros Heterodermia e Polyblastydium. Iheringia 72: 255-266.

Benatti MN, Kitaura MJ, Dias IPRC \& Marcelli MP (2013) Cianoliquens dos gêneros Coccocarpia, Collema e Leptogium do Parque Estadual da Cantareira, SP, Brasil, depositados no herbário SP. Hoehnea 40: 131-141.

Brodo IM, Sharnoff SD \& Sharnoff S (2001) Lichens of North America. Yale University Press, New Haven \& London. 795p.

Calvelo S \& Liberatore S (2002) Catálogo de los líquenes de la Argentina. Kurtziana 29: 7-170.

Esslinger TL \& Egan RS (1995) A sixth checklist of the lichen-forming, lichenicolous, and allied fungi of the continental United States and Canada. The Bryologist 98: 467-549.

Fleig M (1988) Liquens da Estação Ecológica do Taim, Rio Grande, RS, Brasil. Napaea 6: 9-16.

Fleig M \& Grüninger W (2000a) Liquens do Pomar Cisne Branco e arredores, São Francisco de Paula, Rio Grande do Sul, Brasil. Iheringia Série Botânica 53: 67-78.

Fleig M \& Grüninger W (2000b) Levantamento preliminar dos liquens do Centro de Pesquisas e Conservação da Natureza Pró-Mata, São Francisco de Paula, Rio Grande do Sul, Brasil. Napaea 12: 5-20.

Galloway DJ (1985) Flora of New Zealand - lichens. Government Printer, Wellington. 662p.

Galloway DJ (2007) Flora of New Zealand Lichens. Revised Second Edition Including Lichen-Forming and Lichenicolous Fungi. Vol. 1 and 2. Manaaki Whenua Press, Lincoln, New Zealand. 2397p.

Galloway DJ \& Moberg R (2005) The lichen genus Physcia (Schreb.) Michx. (Physciaceae: Ascomycota) in New Zealand. Tuhinga 16: 59-91.

Hale ME (1979) How to know the Lichens. The PicturedKey Nature Series. Dubuque, Iowa: WM. C. Brown Company Publishers, Dubuque. 246p. 
Hale ME (1987) How to know the Lichens. $2^{\text {nd }}$ ed. WCB/ McGraw-Hill, Boston. 246p.

Hansen CJ, Lendemer JC \& Beeching SQ (2008) Contributions to the lichen flora of Alabama: recent collections from four counties. Opuscula Philolichenum 5: 43-48.

Harris RC (1990) Some Florida Lichens. Published by the Author. Bronx, Nova York. 109p.

Huneck S \& Yoshimura I (1996) Identification of Lichen Substances. Springer-Verlag, Berlin, Heidelberg. 493p.

Jungbluth P (2010) Estudos taxonômicos em Physcia (Schreb.) Michx. e Pyxine Fr. Tese de Doutorado. Instituto de São Paulo, São Paulo. 228p.

Jungbluth P \& Marcelli MP (2012) Identificação de Physciaceae s.s. foliosas brasileiras. Glalia 4: $65-$ 101.

Käffer MI \& Mazzitelli SMAM (2005) Fungos liquenizados corticícolas e terrícolas da área da sub-bacia do Sinos e Taquari-Antas, RS, Brasil. Acta Botanica Brasilica 19: 813-817.

Krog H (2000) Corticolous macrolichens of low montane rainforests and moist woodlands of eastern Tanzania. Sommerfeltia 28: 1-75.

Lisboa MA(1952) Lichênes de Ouro Preto. Contribuição à Lichenologia de Ouro Preto apresentada à Terceira Reunião Anual da Sociedade de Botânica do Brasil: 3-8.

Llimona X \& Hladun NL (2001) Checklist of the lichens and lichenicolous fungi of the Iberian Peninsula and Balearic Islands. Bocconea 14: 1-581.

Lynge B (1924) On South America Anaptychiae and Physciae. Kristiania 16: 1-47.

Malcolm WM \& Galloway DJ (1997) New Zeland Lichens: checklist, key and glossary. Museum of New Zeland Te Papa Tongarewa. Wellington. 192p.

McCarthy PM \& Elix JA (2002) Additional lichen records from Australia 48. Miscellaneous taxa in Christmas Island. Australasian Lichenology 50: 10-22.

Marcano V, Morales Méndez A, Sipman HJM \& Calderon L (1996) A first checklist of the lichen-forming fungi of the Venezuelan Andes. Tropical Bryology 12: 193-235.

Marcelli MP (1991) Aspects of the foliose lichen flora of the southern-central coast of São Paulo state, Brazil. In: Galloway DJ (ed.) Tropical lichens: their systematics, conservation, and ecology. Systematics Association Special. Vol. 43. Clarendon Press, Oxford. Pp. 151-170.

Marcelli MP (1998) History and current knowledge of Brazilian lichenology. In: Marcelli MP \& Seaward MRD (eds.) Lichenology in Latin America: history, current knowledge and applications. CETESB, São Paulo. Pp. 25-45.

Marcelli MP(2011) Checklist of lichens and lichenicolous fungi of Brazil. Version 1: June 2004. Disponível em $<$ http://www.biologie.unihamburg.de/checklists/ brazil_1.htm>. Acesso em junho 2011.
Mazzitelli SMAM, Käffer MI \& Cardoso N (1999) Liquens corticícolas de Porto Alegre, Rio Grande do Sul, Brasil. Iheringia, Série Botânica 52: 55-63.

Moberg R (1986) The genus Physcia in East Africa. Nordic Journal of Botany 6: 843-864.

Moberg R (1989) Studies on Physcia. III. Three Physcia species new to Europe and The Azores. Herzogia 8: 249-253.

Moberg R (1990) The lichen genus Physcia in Central and South America. Nordic Journal of Botany 10: 319-342.

Moberg R (1997) The lichen genus Physcia in the Sonoran Desert and adjacent areas. Symbolae Botanicae Upsalienses 32: 163-186.

Moberg R (2001) The lichen genus Physcia in Australia. Bibliotheca Lichenologica 78: 289-311.

Moberg R (2002) Physcia. In: Nash TH, Ryan BD, Gries C \& Bungartz F (eds.) Lichen Flora of the Greater Sonoran Desert Region. I. Lichens Unlimited, Arizona State University, Tempe. Pp. 358-373.

Moberg R (2004) Notes on foliose species of the lichen family Physciaceae in southern Africa. Symbolae Botanicae Upsalienses 8: 257-288.

Moore BJ (1968) The macrolichens flora of Florida. The Bryologist 71: 161-267.

Orange A, James PW \& White FJ (2001) Microchemical methods for the identification of lichens. The British Lichen Society, London. 101p.

Osorio HS (1970) Lichens from Cantera, south Paraguay. Comunicaciones Botánicas Del Museo de Historia Natural de Montevideo 4: 1-3.

Osorio HS (1977) Contribution to the lichen flora of Brazil. II. Lichens from Guarapuaca, Parana state. Dusenia 10: 101-102.

Osorio HS (1981) Contribution to the lichen flora of Brazil VII. Lichens from Morro do Côco, Viamão, Rio Grande do Sul. Phytologia 48: 72-76.

Osorio HS (1992) Contribucion a la flora liquénica del Uruguay. XXV. Lichenes publicados entre 1972 a 1991. Anales del Museo Nacional de Historia Natural de Montevideo 2. serie, Vol. VIII: 43-70.

Osorio HS, Aguiar LW \& Martau L (1997) Contribuição à flora liquênica do Brasil XXXIII. Liquens do estado do Rio Grande do Sul: depressão central. Iheringia 49: 11-20.

Pereira WR \& Marcelli MP (1989) Líquens da Reserva Biológica do Alto da Serra de Paranapiacaba. Acta Botanica Brasilica 3: 89-93.

Scutari NC (1990) Studies on foliose Pyxinaceae (Lecanorales, Ascomycotina) from Argentina. I: anatomical-ontogenetic studies in Physcia tribacia (Ach.) Nyl. Nova Hedwigia 50: 451-461.

Scutari NC (1992) Estudios sobre Pyxinaceae foliosas (Lecanorales, Ascomycotina) de la Argentina, IV: claves de los generos y las especies de la Provincia de Buenos Aires. Boletin de la Sociedad Argentina de Botanica 28: 169-173.

Scutari NC (1995) Los macrolíquenes de Buenos Aires, 
II: Phaeophyscia, Physcia y Pyxine (Physciaceae, Ascomycotina). Darwiniana 33: 21-231.

Sipman HJM \& Wolf JHD (1998) Provisional checklist for the lichens of Chiapas. Acta Botánica Mexicana 45: 1-29.

Swinscow TDV \& Krog H (1988) Macrolichens of East Africa. British Museum of Natural History, London. 390p.

Tenório LU, Sipman HJM \& Lücking R (2002) Preliminary checklist of lichens from Costa Rica. Disponível em < http://archive.fieldmuseum.org/ ticolichen/checklist.html>. Acesso em janeiro 2014.

Thomson JW (1963) The Lichen Genus Physcia in North America. Beihefte zur Nova Hedwigia 7: 1-172.

Vainio EA (1890) Etude sur la classification et la morphologie des lichens du Brésil, I. Acta Societatis pro Fauna et Flora Fennica 7: 1-247.

Walker JW \& James PW (1980) A revised guide to microchemical techniques for the identification of lichen products. Bulletin of the British Lichen Society 46 (supl.) London: 13-29.

White FJ \& James PW (1985) A new guide to microchemical techniques for the identification of lichen substances. Bulletin of the British Lichen Society 57 (supl.) London: 1-41.

Wietzke-Beckenkamp L \& Pereira AB (1997) Liquens corticícolas do Parque da Gruta, Santa Cruz do Sul-RS, Brasil. Logos 9: 81-92.

Wolseley PA, Aguirre-Hudson B \& McCarthy P (2002) Catalogue of the lichens of Thailand. Bulletin of the Natural History Museum, London (Bot.) 32: 13-59.

Zahlbruckner A (1909) Lichenes (Flechten). In: Schiffner V (ed.) Ergebnisse der botanischen Expedition der kaiserlichen Akademie der Wissenschaften nach Südbrasilien, 1901, Band 2. Denkschriften der Kaiserlichen Akademie der Wissenschaften 83: 85-211. 